\title{
Relative Content Evaluation of Single-walled Carbon Nanotubes using UV-VIS-NIR Absorption Spectroscopy
}

\author{
Ok Hwan Cha ${ }^{1}$, Mun Seok Jeong ${ }^{1, \wedge}$, Clare C. Byeon ${ }^{1}$, Hyun Jeong ${ }^{2}$, Jong Hun $\mathrm{Han}^{3}$, Young Chul \\ $\mathrm{Choi}^{4}$, Kay Hyeok An ${ }^{5}$, Kyunghui $\mathrm{Oh}^{6}$, Ki Kang $\mathrm{Kim}^{7}$ and Young Hee Lee
}

\author{
${ }^{1}$ Advanced Photonics Research Institute, Gwangju Institute of Science and Technology, Gwangju, 500-712, Korea \\ ${ }^{2}$ Department of Semiconductor Science and Technology, Chonbuk National University, Jeonju, 561-756, Korea \\ ${ }^{3}$ Energy \& Nanomaterials Research Center, Korea Electronics Technology Institute, Seongnam 463-816, Korea \\ ${ }^{4}$ R\&D Center, Hanwha Nanotech, 423-1 CheongCheon-dong, Bupyeong-gu, Incheon, 403-030, Korea \\ ${ }^{5}$ Material \& Development Department, Jeonju Machinery Research Center, Jeonju 561-844, Korea \\ ${ }^{6}$ Korean Agency for Technology and Standards, Gwacheon, 427-723, Korea
}

${ }^{7}$ BK 21 Physics Division, Institute of Basic Science, Center for Nanotubes and Nanostructured Composites, Sungkyunkwan University, Suwon 440-746, Korea ‘e-mail: mjeong@gist.ac.kr

(Received February 16, 2009; Accepted March 14, 2009)

\begin{abstract}
We propose an evaluation method of the relative content of single-walled carbon nanotubes (SWCNT) in SWCNT soot synthesized by arc discharge using UV-VIS-NIR absorption spectroscopy. In this method, we consider the absorbance of semiconducting and metallic SWCNTs together to calculate the relative content of SWCNTs with respect to a highly purified reference. Our method provides the more reliable and realistic evaluation of SWCNT content with respect to the whole carbonaceous content than the previously reported method.
\end{abstract}

Keywords : Carbon nanotube, Purity measurement, UV-VIS-NIR absorption

\section{Introduction}

Since its discovery in 1991, the carbon nanotube (CNT) has become an important material of interest in various fields due to its unique physical and chemical properties [1]. Some challenging applications of CNT have been proposed or realized in many new functional materials and devices such as various emitters, field emission display (FED), contacts in $\mathrm{Li}$ ion secondary battery, hydrogen storages, nanowires, AFM/STM tips, single electron transistor, gas sensors, etc [2-4].

Despite of much enthusiasm of utilizing CNTs in many research fields, its commercialization for industrial products is not yet as blooming as in basic and application research. One of the main reasons for this holding back is due to the fact that there is no standard definition or the method of content evaluation globally accepted. Each manufacturer uses its own method of evaluating the content of CNTs and the different methods of evaluation distress the developers that the consistency of their outcomes is at risk, causing a critical delay in development of new applications and commercialization. Thus, a globally standardized method of evaluating the content of CNTs is desperately demanded not only from science communities but also from industry [5-10].

The major portion of impurities and debris occasionally produced during single-walled carbon nanotube (SWCNT) synthesis is carbonaceous, such as amorphous carbons and carbon nanoparticles. Transmission electron microscopy (TEM) and scanning electron microscopy (SEM) are the typical tools for analyzing qualitatively the content of these carbonaceous impurities. Compared with TEM, SEM is easier to operate and requires simpler pre-processing procedures for the [7-9]. Even though SEM is a very reliable way of directly observing the presence of the carbonaceous impurities in SWCNT, however, the amount of SWCNT that can be detected in a single scanning is only about one trillionth of a gram. This limits SEM from being used for evaluating the content of a large amount of SWCNT in grams, which most of SWCNT vendors offer. On the contrary, the optical methods provide a relatively fast way of evaluating a large amount of SWCNT [7-9]. Among the various optical methods, Raman scattering is used for qualitative evaluation, and absorption spectroscopy is used for quantitative measurement. Itkis et al. previously suggested a method based on absorption spectroscopy for evaluating the content of SWCNT [7], which requires very simple sample preparations and costs only one tenth of SEM. Nevertheless, this method only measures the content of semiconducting SWCNT as whole from the absorption characteristics, assuming no metallic SWCNT presence. 
Thus, the method may not be reliable for evaluating the content of SWCNT soot containing both semiconducting and metallic SWCNT. Ryabenko et al. also suggested another method, but the method measures the absorption of metallic SWCNT only [8]. More recently, Landi et. al. made an attempt of evaluating the content of SWCNT by removing the -plasmon peak from the absorption spectrum following Lorentzian curve-fitting. However, this method also has a questionable accuracy [9].

In this Letter, we propose a new method of evaluating the content of SWCNTs using UV-VIS-NIR absorption spectroscopy which considers the amounts of both semiconducting and metallic SWCNTs in SWCNT soot.

\section{Experimental}

With current technology, a $100 \%$ SWCNT reference, required to To measure the absolute amount of SWCNTs in SWCNT soot, is not practically available. In our study, a highly purified SWCNT sample is prepared instead by purifying SWCNT soot and used for all our measurements as a reference. It should be noted that the relative content of SWCNTs that we measured here will change when the highly purified reference with even higher purity is available in future. The reference sample was synthesized by arc-discharge with iron sulfide (FeS), nickel (Ni), and cobalt (Co) catalysts followed by several repetitive procedures of thermal annealing at $350^{\circ} \mathrm{C}$ for 30 minutes in the air, nitric acid and hydrochloric acid treatment and centrifugation. Fig. 1 (a) shows a SEM image of the highly purified reference SWCNT. The SEM image does not show the presence of carbonaceous impurity, verifying the high purity of the reference. Fig. 1 (b) shows UV-VIS-NIR absorption spectrum of the reference. In the figure, semiconducting $\left(\mathrm{S}_{11}, \mathrm{~S}_{22}\right)$, metallic $\left(\mathrm{M}_{11}\right)$, and $\pi$ plasmon peaks of SWCNT are clearly visible. The purity value calculated from the absorption spectrum curve using the method suggested by Itkis et al. was about 0.23 [7]. The reference SWCNT was purified until the amount of noncarbonaceous impurity was below $1 \%$ detected by thermogravimetric analysis(TGA) as shown in Fig. 1 (c).

The procedures for preparing a test sample for content evaluation are described as follows. The water content in the test SWCNT sample was removed by heating up for 12 hours at $200^{\circ} \mathrm{C}$ with a continuous flow of nitrogen gas $\left(\mathrm{N}_{2}: 99.999 \%\right) .1 \mathrm{mg}$ of the dried SWCNT sample was then placed in a $100 \mathrm{ml}$ container. A SWCNT solution was made by adding $100 \mathrm{~mL}$ of $\mathrm{N}$ - dimethylformamide (DMF: $99.8 \%$ with less than $0.005 \%$ water). The SWCNT solution was sonicated for 6 hours for dispersion. During the sonication, the water level of ultrasonicator was kept higher than that of the SWCNT solution in the $100 \mathrm{~mL}$ container. The ultrasonicator was working at $40 \mathrm{kHz}$ and $240 \mathrm{~W}$. No other apparatus was in the water container of ultrasonicator, and
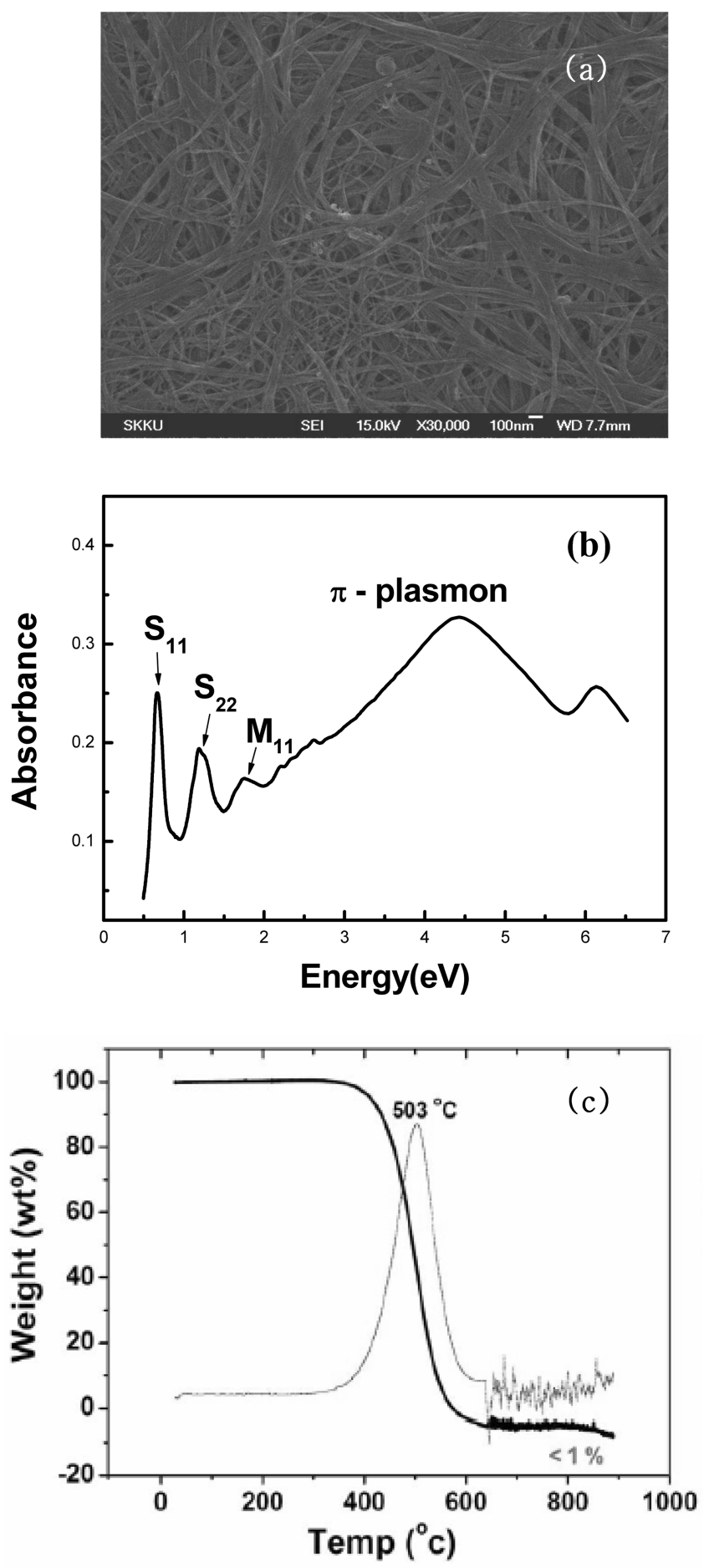

Fig. 1. (a) SEM image, (b) Absorbance, and (c) TGA data of highly purified SWCNT to be used as a reference.

water was constantly added during the sonication for it might cause the temperature change. A series of SWCNT samples were prepared in $10 \%$ interval in content from 0 to $100 \%$ by adding carbon nanoparticles to the purified reference accordingly [9]. 

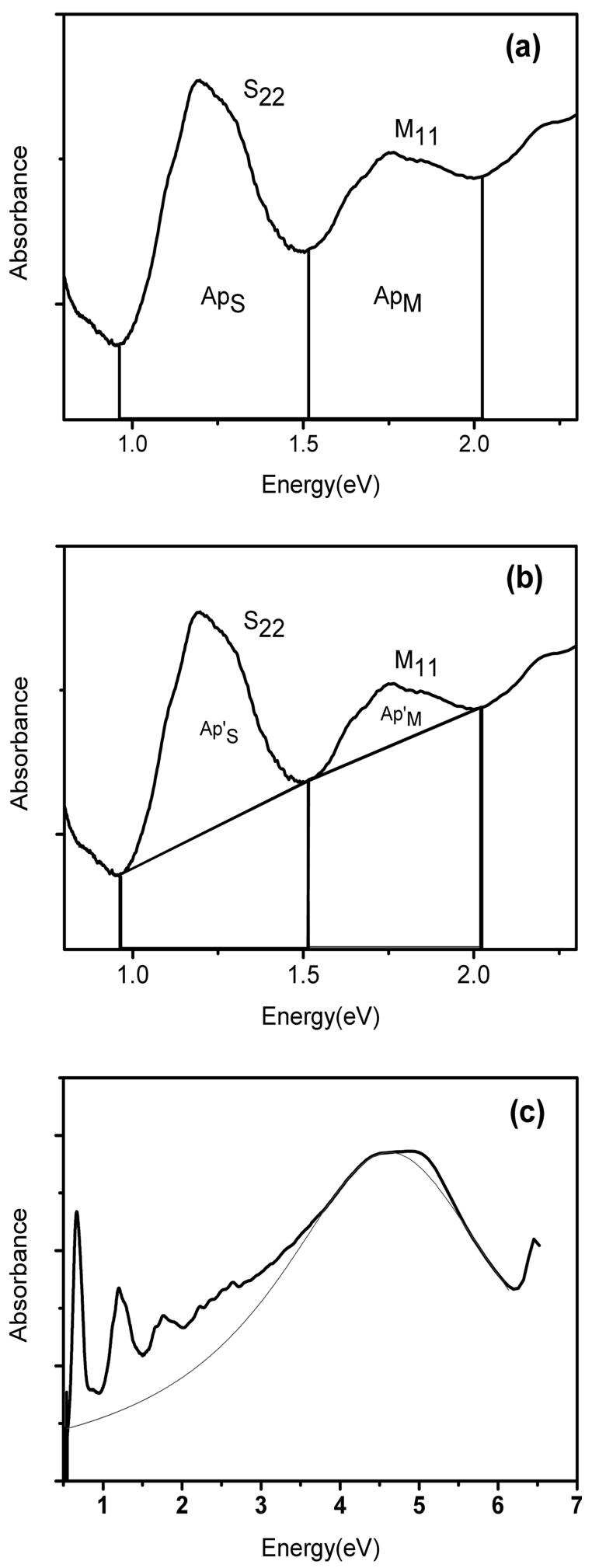

Fig. 2. Calculation method of relative content of SWCNTs from the absorbance; (a) Integrated spectrum, (b) Integrated spectrum by modified baselines for removing the $\pi$-plasmon effect, and (c) estimation of the $\pi$-plasmon peak using nonlinear fitting method suggested by Landi et.al. [9].
Absorption spectroscopy was performed with following conditions. The reference SWCNT solution, assumed to be $100 \%$ SWCNT, and the SWCNT solutions to be evaluated were placed each in a $1 \mathrm{~mm}$ path length spectroscopic quartz cell, and the absorption spectrum was measured using a UVVIS-NIR spectrophotometer (V-570, JASCO). The SWCNT was placed in the cell prior to addition of the dispersion solution when the solution was mixed. The cell was always capped to minimize the solvent evaporation. The average value was taken out of three trials.

\section{Results and Discussion}

Fig. 2 shows some guiding lines of calculating the amount of SWCNTs in SWCNT soot from the absorption spectrum curve. As Itkis and Landi reported, $\mathrm{S}_{11}$ peak is very sensitive to the doping condition and environment because it is near Fermi level [7,9]. So, $\mathrm{S}_{22}$ and $\mathrm{M}_{11}$ peaks, which are further away from Fermi level, were used in evaluation. A previous method by Itkis et al. calculated the relative content of SWCNT by comparing the integrated absorbance of $\mathrm{S}_{22}$ peak to that of the $S_{22}$ peak of the highly purified reference, assuming the contribution of $\pi$-plasmon was constant throughout all spectral region of interest [7]. Ryabenko et al. only used the $\mathrm{M}_{11}$ peak for evaluating the SWCNT content [8]. More recent researches have been focusing on separation of semiconducting SWCNTs and metallic SWCNTs. It is well known that the ratio of semiconducting SWCNTs and metallic SWCNTs takes a variety of different values depending on synthesizing methods and chemical processing. Consequently, calculating the amount of SWCNTs in SWCNT soot taking only one chirality into account would cause a problem in accuracy [8,11-17]. Landi et al. suggested a method of including both semiconducting and metallic peaks [9]. This method used the absorption spectrum curve only in $4.0 \sim 4.25 \mathrm{eV}$ because the full spectral observation of $\pi$-plasmon peak was difficult due to the absorption in chemical solution. The energy, intensity, and width of the $\pi$-plasmon peak were estimated and then the $\pi$ plasmon peak was removed by nonlinear fitting in calculating the amount of SWCNTs. Thus, the content value varies depending on the fitting parameters even when the fitting agrees with the measurement with $99 \%$ accuracy in $4.0 \sim 4.25 \mathrm{eV}$.

For this reason, we applied the linear fitting method suggested by Itkis et al. to $\mathrm{S}_{22}$ and $\mathrm{M}_{11}$ peaks including both semiconducting and metallic SWCNT in evaluating the content as follows [7,9]. First, we integrated the $S_{22}$ peak and $\mathrm{M}_{11}$ peak in absorption spectrum of the reference SWCNT solution, (Fig. 2 (a)) labeled each integration value as $A p_{S}$, and $A p_{M}$ respectively, and labeled the sum of these two values as $A p_{S M}$. Then, a straight line was drawn between each end point of the $S_{22}$ peak as in Fig. 2 (b). The area between 


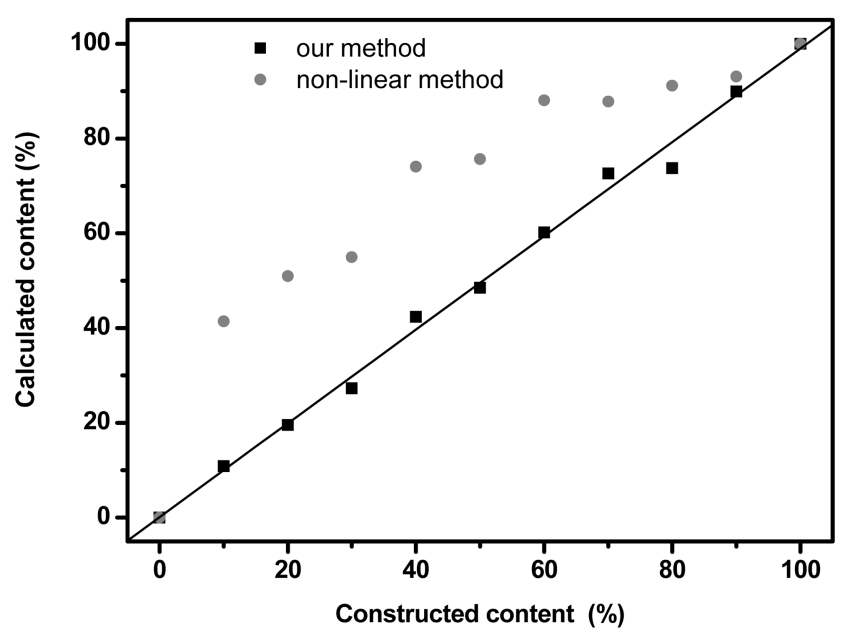

Fig. 3. Evaluated content vs. Constructed content of SWCNTs calculated with our method(square) and non-linear method (circle).

the spectrum curve and the straight line was calculated, and labeled as $A p_{S}^{\prime}$. The same procedure was performed for the $\mathrm{M}_{11}$ peak, and the area was labeled as $A p^{\prime}{ }_{M}$. The sum of $A p_{S}^{\prime}$ and $A p_{M}^{\prime}$ was labeled as $A p_{S M}^{\prime} . A p_{S M}^{\prime}$ was then divided by $A p_{S M}$, and the quotient was labeled as $P_{S M}$.

Next, we integrated the $S_{22}$ peak and $M_{11}$ peak in absorption spectrum of the SWCNT solution to be evaluated, labeled each value as $A t_{S}$ and $A t_{M}$ respectively, and labeled the sum of these two values as $A t_{S M}$. A straight line was drawn between each end point of the $\mathrm{S}_{22}$ peak. The area between the spectrum curve and the straight line was calculated, and labeled as $A t_{S}^{\prime}$. The same procedure was performed for the $\mathrm{M}_{11}$ peak, and the area was labeled as $A t_{M}^{\prime}$. The sum of $A t_{S}^{\prime}$ and $A t_{M}^{\prime}$ was labeled as $A t_{S M}^{\prime} . A t_{S M}^{\prime}$ was then divided by $A t_{S M}$, and the quotient was labeled as $T_{S M}$.

To calculate the content of SWCNT in SWCNT soot, the following formula was applied.

Content of the single-walled carbon nanotubes $(\%)=\frac{T_{S M}}{P_{S M}} \times 100$ where $T_{S M}=\frac{A T_{S_{M}}^{\prime}}{A t_{S M}}=\frac{A t^{\prime}{ }_{S}+A t^{\prime}{ }_{M}}{A t_{S}+A t_{M}}$ and

$$
P_{S M}=\frac{A p^{\prime}{ }_{S M}}{A p_{S M}}=\frac{A p^{\prime}{ }_{S}+A p^{\prime}{ }_{M}}{A p_{S}+A p_{M}}
$$

We applied the method to the series of SWCNT samples with intentionally varied content, and the results are shown in Fig. 3. Fig. 3 plotted the evaluated content (squares) from the measurement to the constructed content with varying content from 0 to $100 \%$. The linear fitting yielded a slope very close to unity, 0.998 , and the confidence value $\mathrm{R}^{2}$ was 0.994. For comparison, we also plotted in Fig. 3 the content calculated from removing the $\pi$-plasmon peak by nonlinear fitting as Landi et. al. did (circles). As shown in Fig. 3, the method provided by Landi et al. yields quite significant discrepancies from the presumably true values of contents. On the contrary, our evaluation results of the SWCNT content are in excellent agreement with the designed values of constructed contents.

In this study, we only used the SWCNTs synthesized by arc discharge method. We believe that our method would produce the similar result with a reasonable accuracy in content evaluation also for the SWCNTs synthesized by HipCo or laser vaporization.

\section{Conclusions}

We have suggested a method of evaluating the content of SWCNT among the carbon complexes in SWCNT soot using UV-VIS-NIR absorption spectroscopy. We have verified the result of our method using a series of constructed samples with designed content that the evaluated contents are well matched with high enough accuracy to the designed contents from the purified reference. We believe that our method provides an accurate way to evaluate the content of SWCNTs and may play a key role in investigating the relationship between the purity of SWCNT to its functionality in many applications.

\section{Acknowledgment}

This work was supported by Korean Agency for Technology and Standards and by APRI-Research Program through a grant provided by Gwangju Institute of Science and Technology in 2009.

\section{References}

[1] a) Iijima, S. Nature, 1991, 354, 56. b) Iijima, S.; Ichihashi, T. Nature 1993, 363, 603.

[2] Baughman, R. H.; Zakhidov, A. A.; de Heer, W. A. Science 2002, 297, 787

[3] Fan, S.; Chapline, M. G. N.; Franklin, R.; Tombler, T. W.; Cassell, A. M.; Dai, H. Science 1999, 283, 512.

[4] a) Dresselhaus, M. S.; Dresselhaus, G.; Eklund, P. C. "Science of Fullerenes and Carbon Nanotubes", Academic Press, San Diego, 1996, 689. b) Sharon, M.; Rusop, M.; Soga T.; Afre, R. A. Carbon Lett. 2008, 9, 17.

[5] Thess, A.; Lee, R.; Nikolaev, P.; Dai, H. J.; Petit, P.; Robert, J.; Xu, C. H.; Lee, Y. H.; Kim, S. G.; Rinzler, A. G.; Colbert, D. T.; Scuseria, G. E.; Tomanek, D.; Fischer, J. E.; Smalley, R. E. Science 1996, 273, 483.

[6] Kong, J.; Cassell, A. M.; Dai, H. J. Chem. Phys. Lett. 1998, 292, 567.

[7] Itkis, M. E.; Perra, D. E.; Niyogi, S.M.; Hamon, M. A.; Hu, 
H.; Zhao, B.; Haddon, R. C. Nano Lett. 2003, 3, 309.

[8] Ryabenko, A. G.; Dorofeeva, T. V.; Zvereva, G. I. Carbon 2004, 42, 1523

[9] Landi, B. J.; Herbert, J. R.; Evans, C. M.; Cress, C. D.; Raffaelle, R. P. J. Phys. Chem. B 2005, 109, 9952.

[10] Park, Y. S.; Kim, K. S.; Jeong, H. J.; Kim, W. S.; Moon, J. M.; An, K. H.; Bae, D. J.; Lee, Y. S.; Park, G.-S.; Lee, Y. H. Synth. Met. 2004, 140, 1.

[11] Krupke, R.; Hennrich, F.; Lohneysen, H. V.; Manfred, M.; Kappes, M. M. Science 2003, 301, 344.

[12] Zheng, M.; Jagota, A.; Strano, M. S.; Santos, A. P.; Barone, P.; Chou, S. G.; Diner, B. A.; Dresselhaus, M. S.; Mclean, R. S.; Onoa, G. B.; Samsonidze, G. G.; Semke, E.
D.; Usrey, M.; Walls, D. J. Science 2003, 302, 1545.

[13] Chattopadhyay, D.; Galeska, I.; Papadimitrakopoulos, F. J. Am. Chem. Soc. 2003, 125, 3370.

[14] Chen, Z.; Du, X.; Du, M.-H.; Rancken, C. D.; Cheng, H.P.; Rinzler, A. G. Nano Lett. 2003, 3, 1245.

[15] An, K. H.; Park, J. S.; Yang, C.-M.; Jeong, S. Y.; Lim, S. C.; Kang, C.; Son, J.-H.; Jeong, M. S.; Lee, Y. H., J. Am. Chem. Soc. 2005, 127, 5196.

[16] An, K. H.; Lee. Y. H. NANO 2006, 1, 115.

[17] Maeda, Y.; Hashimoto, M.; Hasegawa, T.; Kanda, M.; Tsuchiya, T.; Wakahara, T.; Akasaka, T.; Miyauchi, Y.; Maruyama, S.; Lu, J.; Sagase, S., Nano 2007, 2, 221. 